\title{
In Vitro Communities Derived from Oral and Gut Microbial Floras Inhibit the Growth of Bacteria of Foreign Origins
}

\author{
Xuesong He • Yan Tian • Lihong Guo • Takashi Ano • \\ Renate Lux • David R. Zusman • Wenyuan Shi
}

Received: 7 February 2010 / Accepted: 16 June 2010 / Published online: 13 July 2010

(C) The Author(s) 2010. This article is published with open access at Springerlink.com

\begin{abstract}
The gastrointestinal (GI) tract is home to trillions of microbes. Within the same GI tract, substantial differences in the bacterial species that inhabit the oral cavity and intestinal tract have been noted. While the influence of host environments and nutritional availability in shaping different microbial communities is widely accepted, we hypothesize that the existing microbial flora also plays a role in selecting the bacterial species that are being integrated into the community. In this study, we used cultivable microbial communities isolated from different parts of the GI tract of mice (oral cavity and intestines) as a model system to examine this hypothesis. Microbes from these two areas were harvested and cultured using the same nutritional conditions, which led to two distinct microbial communities, each with about 20 different species as revealed by
\end{abstract}

Electronic supplementary material The online version of this article (doi:10.1007/s00248-010-9711-9) contains supplementary material, which is available to authorized users.

X. He $\cdot$ Y. Tian $\cdot$ R. Lux $\cdot$ W. Shi $(\bowtie)$

UCLA School of Dentistry,

10833 Le Conte Avenue, CHS 20-114,

Los Angeles, CA 90095-1668, USA

e-mail:wenyuan@ucla.edu

L. Guo $\cdot$ W. Shi

Department of Oral Biology,

Peking University School and Hospital of Stomatology,

22 Zhong Guan Cun Nan Da Jie, Haidian District,

Beijing 100081, China

T. Ano

Department of Biotechnological Science, Kinki University,

Kinokawa City, Wakayama 649-6493, Japan

D. R. Zusman

Department of Molecular and Cell Biology,

University of California,

Berkeley, CA 94720, USA
PCR-based denaturing gradient gel electrophoresis analysis. In vitro community competition assays showed that the two microbial floras exhibited antagonistic interactions toward each other. More interestingly, all the original isolates tested and their closely related species displayed striking community preferences: They persisted when introduced into the bacterial community of the same origin, while their viable count declined more than three orders of magnitude after 4 days of coincubation with the microbial flora of foreign origin. These results suggest that an existing microbial community might impose a selective pressure on incoming foreign bacterial species independent of host selection. The observed inter-flora interactions could contribute to the protective effect of established microbial communities against the integration of foreign bacteria to maintain the stability of the existing communities.

\section{Introduction}

Gastrointestinal (GI) tract-associated indigenous microbiota often co-evolve with their hosts and has been shown to play important roles in the host's health and disease $[18,19,26]$. Classical 16S rRNA gene sequence analyses and recent metagenomic studies in the mouse and human GI tract microbiomes revealed complex ecosystems comprised of large numbers of microbial species, including bacteria, archaea, yeast, and fungi, with bacteria being the most dominant residents [1, 12, 30, 31, 39, 41, 42].

The human intestinal microbiota contains thousands of different bacterial species. This diverse community is a very important part of the GI tract and has been regarded as an essential "organ." It carries critical functions in digesting unutilized substrates [37], training the immune system [7], protecting against epithelial cell injury [32], and repressing pathogenic microbial growth [14]. On the other hand, the 
intestinal microbiota has been suggested to contribute to pathogenic conditions, such as cancer [14] and inflammatory bowel disease $[15,22]$. Additionally, the intestinal microbial flora has been linked to certain persistent health conditions including obesity [20].

The human oral cavity is an important part of the GI tract which harbors more than 700 bacterial species and thereby represents another very complex endogenous microbial flora $[1,17,29]$. The study of the oral cavity-associated microbial flora has been predominantly focused on their implication on oral diseases such as dental caries and periodontitis [10, 23, $26,36]$. More recently, a number of oral species have been suggested to be involved in a number of chronic diseases, including cardiovascular diseases [2], osteomyelitis [11], and bacterial endocarditis [3]. Although beneficiary roles have been suggested for the oral microflora, conclusive evidence remains to be provided.

Even though both oral cavity and intestinal tract are parts of the same GI tract and most, if not all, of the GI tractassociated microbes are initially introduced through the oral cavity [28], recent community studies have noted substantial differences in the bacterial species that inhabit the oral cavity and intestinal tract $[25,29,31]$. One of the fundamental questions regarding the GI tract-associated microbiota is how microbial communities are formed and maintained [19]. The current concept proposes that the establishment of endogenous microbial flora is mainly shaped by the following two effects: (1) the legacy effect, which refers to the inheritance of microflora from a parent, or the microbial composition in the local environment [19] and (2) the host habitat effect, which is the distinct selective pressure derived from the specific micro-environment within the hosts, such as their anatomical structures, available nutrients, and local immune properties [33].

In this study, we hypothesize that the existing hostassociated microflora contributes to its own shaping and maintenance by selecting the bacterial species that are being integrated into the community ("community selection" effect). To test this hypothesis, we established an in vitro experimental system with microbial communities derived from the oral cavity and intestines of mice under the same nutritional conditions to minimize the legacy and host habitat effects. These in vitro model communities were used to study the interactions between the oral and gut microbial floras.

\section{Materials and Methods}

Preparation of BHI-Cultivable Microbial Flora from Mice Oral and Intestinal Samples

Five male C57BL/6 mice were kept individually in cages with wood chips bedding. They were fed a commercial diet
(Halan-Teklad Ltd., Madison, WI, USA) and distilled water ad libitum. Mice were sacrificed at 20 weeks of age. The oral cavity was rinsed twice with $500 \mu \mathrm{l}$ phosphatebuffered saline (PBS) buffer; the mandible with two incisors and six molars were removed and immersed in $1.5 \mathrm{ml}$ of PBS buffer containing the previous oral rinse. Ultra-sonication (power output $60 \mathrm{~W}$ ) was applied for $20 \mathrm{~s}$ to disperse the attached microorganisms, and samples from the five animals were pooled. The cecum contents of the five mice were collected, pooled, and resuspended in PBS buffer.

Various rich media were evaluated and brain heart infusion (BHI) medium (Difco) supplemented with hemin $(5 \mu \mathrm{g} / \mathrm{ml})$, vitamin $\mathrm{K}(0.5 \mu \mathrm{g} / \mathrm{ml})$, sucrose $(0.1 \%)$, mannose $(0.1 \%)$, and glucose $(0.1 \%$; simply referred to as $\mathrm{BHI}$ in this study) was the final choice as it was able to support the growth of both oral and intestinal bacterial communities with similar high microbial diversity (refer to result section). Cultures were incubated at $37^{\circ} \mathrm{C}$ under microaerobic conditions (nitrogen $90 \%$, carbon dioxide 5\%, oxygen 5\%) until turbid. Frozen stocks for each cultured flora were prepared by adding glycerol to the samples to a final concentration of $25 \%$. Samples were stored at $-80^{\circ} \mathrm{C}$ as the stock of BHI-cultivable oral cavity and intestinal microbial floras.

Isolation and Identification of Bacterial Species from Oral and Intestinal Samples

Stocks of BHI-cultivable oral and intestinal microbial flora were diluted in BHI medium and seeded on BHI agar plates supplemented with hemin and vitamin $\mathrm{K}$. The plates were incubated for 5 days at $37^{\circ} \mathrm{C}$ under microaerobic conditions. Potentially different bacterial species were picked from the plate based on their differences in morphology, pigmentation, and the incubation time for colonies to appear. Individual colonies were grown in supplemented $\mathrm{BHI}$ at $37^{\circ} \mathrm{C}$ under microaerobic conditions until turbid. Bacterial cells were collected, and genomic DNA of each isolate was prepared using the MasterPure ${ }^{\mathrm{TM}}$ DNA purification kit (EPICENTRE, Madison, WI, USA).

For species identification, the universal bacterial $16 \mathrm{~S}$ rDNA primer pair, $27 \mathrm{~F}$ and $1492 \mathrm{R}$ [24], was used to generate an approximately 1,500-bp amplicon. Each $50 \mu \mathrm{l}$ PCR reaction mixture contained $20 \mathrm{ng}$ of genomic DNA, $200 \mu \mathrm{M}$ of each dNTP, $4.0 \mathrm{mM} \mathrm{MgCl} 2,100 \mathrm{nM}$ of each primer, $5 \mu \mathrm{l}$ of $10 \times \mathrm{PCR}$ buffer, and $2.5 \mathrm{U}$ of Taq polymerase (Invitrogen). PCR conditions were as follows: 3 min at $94^{\circ} \mathrm{C}$ for initial denaturation and 27 cycles of $94^{\circ} \mathrm{C}$ for $1 \mathrm{~min}, 50^{\circ} \mathrm{C}$ for $1 \mathrm{~min}$, and $72^{\circ} \mathrm{C}$ for $2 \mathrm{~min}$ and a final chain elongation at $72^{\circ} \mathrm{C}$ for $5 \mathrm{~min}$. PCR products were purified using the QIAquick PCR purification kit (Qiagen) and sequenced at the UCLA Core DNA Sequencing 
Facility. Obtained sequences were subjected to BLAST searches against the NCBI and ribosomal database. Altogether, 10 and 11 different bacterial species were isolated from oral and intestinal samples, respectively.

\section{Community Competition Assay}

Original microbial mixtures cultivated from mice were used to establish in vitro intestinal (I-mix) and oral (O-mix) communities. Since biofilms are the most prevalent mode of microbial life in most settings, we attempted to grow both oral and intestinal bacterial communities in their biofilm forms. However, under the conditions we tested, the oral microbial flora formed biofilms, while intestinal flora was unable to do so. We therefore followed a previously described model system in which bacteria are pelleted [16] to most closely mimic biofilm-like conditions and enable interspecies interactions. To confirm that these pelleted communities exhibit similar traits as biofilm grown communities, we compared both conditions for the oral community and found no significant difference in community composition and foreign flora exclusion behavior.

1. Establishing the In Vitro Intestinal Community (I-mix): An overnight culture of the original BHI-cultivable intestinal microbial mixture isolated in this study was diluted 1:100 into fresh supplemented BHI medium and distributed into $15-\mathrm{ml}$ conical tubes. The cultures were incubated at $37^{\circ} \mathrm{C}$ under microaerobic condition until the cell density reached an $\mathrm{OD}_{600}$ of about 1 . Cells were harvested and diluted to an $\mathrm{OD}_{600}$ of 0.5 into fresh medium, and $1.5 \mathrm{ml}$ of the diluted culture was pelleted again by centrifugation at $3,000 \times g$ for $1 \mathrm{~min}$ to form cell pellets. Tubes with cell pellets were incubated microaerobically at $37^{\circ} \mathrm{C}$ overnight to allow establishment of the in vitro intestinal microbial community. For time course experiments, multiple tubes with identical intestinal communities were inoculated at the same time, and individual samples were processed at each time point.

2. Establishing the In Vitro Oral Community (O-mix): An overnight culture of the oral microbial mixture was diluted 1:100 into fresh supplemented BHI medium, and $100 \mu \mathrm{l}$ of the bacterial suspension containing about $10^{6}$ cells were seeded into six-well plates with $1.5 \mathrm{ml}$ supplemented BHI medium in each well. Plates were incubated overnight at $37^{\circ} \mathrm{C}$ under microaerobic conditions to allow biofilm formation of the oral microbial community. Multiple wells with identical oral communities were inoculated, and the complete sample from one well was processed at each time point. Alternatively, the oral microbial community was allowed to establish in pellet form as indicated in the previous section for the intestinal microbial community to determine if the community composition is affected by the mode of growth (biofilm vs pellet).

To examine community level competition, the respective foreign flora was then added at an equal number to the preestablished communities described above. To determine bacterial numbers of the pre-established communities, CFU counts were performed on samples inoculated under the same experimental conditions 1 day prior to setting up the experimental communities. Based on these CFU counts, I-mix and O-mix overnight cultures were adjusted to an equal number in $1.5 \mathrm{ml}$ fresh supplemented BHI and added to the relevant foreign community. Cultures were incubated at $37^{\circ} \mathrm{C}$ microaerobically, and samples were collected every $48 \mathrm{~h}$ over a 4-day time period. To account for the entire community, the biomass of the biofilms was meticulously scraped off the bottom of the well using a sterile spatula. The resulting suspension containing the detached biofilm cells as well as the unattached cells from the supernatant was transferred into a sterile tube. Cells collected from biofilm and pellet experimental setups were spun down at $14,000 \times g$ for $5 \mathrm{~min}$. Samples were treated with ethidium monoazide bromide (EMA) prior to DNA isolation using the MasterPure ${ }^{\mathrm{TM}}$ DNA purification kit (EPICENTRE, Madison, WI, USA) to minimize the impact of non-viable cells prior to PCR-based denaturing gradient gel electrophoresis (PCR-DGGE) analysis. Two biological replicates were performed for each assay.

\section{Community Integration Assay}

The original strains isolated from the mice oral and intestinal communities and closely related bacterial strains carrying antibiotic resistance markers to allow enumeration on selective plates were examined in the community integration assay.

The following two methods were employed to monitor the ability of selected species to integrate into pre-existing communities:

1) PCR-DGGE analysis. Overnight cultures of individual oral (Staphylococcus epidermidis-OI100 and Streptococcus salivarius-OI101) or intestinal (Enterococcus faecalis-II100 and Lactobacillus animalis-II101) strains isolated in this study were adjusted to an $\mathrm{OD}_{600}$ of about 1 and added to the pre-established oral or intestinal microbial communities as described above but at a 1:10 specific species-to-community ratio in cell numbers. Co-cultures were incubated over a 6-day time period, and samples were collected every 2 days by centrifugation. The harvested samples were treated with EMA prior to total genomic DNA isolation for further PCR-DGGE analysis to monitor 
the status of the isolates within the bacterial community. Two biological replicates were performed for each assay.

2) Viability count. To obtain quantitative data, a $S$. salivarius strain (S. salivarius 57.I-AureC::kan) [8] carrying a kanamycin resistance marker and an $E$. faecalis strain (E. faecalis OG1SSp) [9] carrying a spectinomycin resistance marker were used as representative oral and intestinal bacterial species in the community integration assay as described above. Although they were not original species isolated in this study, these two strains were closely related to isolated oral ( $S$. salivarius-OI101) and intestinal (E. faecalis-II100) strains. Co-cultivation samples were taken periodically as described, subjected to serial dilution and plated onto selective and non-selective supplemented BHI agar plates. Plates were incubated for 4 days at $37^{\circ} \mathrm{C}$ under microaerobic condition before colonies were counted. Three replicate experiments were performed.

Examination of Species Composition Required for Inhibitory Effect

Overnight cultures of the four most abundant original oral isolates, including Staphylococcus aureus, Lactobacillus murinus, S. salivarius, and S. epidermidis, as well as the original O-mix were used to establish biofilms containing either mono-species (Streptococcus oralis or L. murinus), defined multi-species (S. oralis, L. murinus, S. salivarius, and $S$. epidermidis), or the whole cultivable oral mix following the procedures described above. E. faecalis OG1SSp was used as a representative of intestinal bacterial species to test the inhibitory effect exerted by each type of biofilm. E. faecalis OG1SSp was added to the pre-formed biofilms in a 1:10 cell number ratio.

Similarly, overnight cultures of selected original intestinal isolates, including L. animalis, E. faecalis, Escherichia coli, and Bacteroides caccae, as well as the original intestinal mix were used to establish pellet communities as described above containing either selected mono-species (L. animalis and $E$. faecalis), defined multi-species ( $L$. animalis, E. faecalis, E. coli, and B. caccae), or the whole cultivable intestinal mix. The oral representative $S$. salivarius strain ( $S$. salivarius 57.I-AureC::kan) carrying a kanamycin resistance marker was added to pre-formed intestinal community in a 1:10 cell number ratio.

Co-cultures for both setups were incubated for a total time period of 4 days. Samples were taken every 2 days, subjected to serial dilution, and plated onto selective and non-selective supplemented BHI agar plates. Plates were incubated for 4 days at $37^{\circ} \mathrm{C}$ under microaerobic condition before colonies were counted. Three replicates were performed for each assay

\section{Spent Medium Assay}

Oral and intestinal microbial communities were established as described above (as biofilm or pellet for oral and intestinal community formation, respectively). After $48 \mathrm{~h}$ of incubation, bacterial cells were pelleted, and supernatants (spent medium) were collected and filter-sterilized. The oral (S. salivarius-OI101) and intestinal (E. faecalisII100) strains isolated in this study were inoculated into both oral and intestinal spent medium, and viability counts were monitored for both strains in the two different spent media every $48 \mathrm{~h}$. Three replicates were performed for each assay.

\section{EMA Cross-linking}

To prevent amplification of DNA from dead bacterial cells and limit DNA-based PCR-DGGE community analysis to the viable fraction, the collected bacterial samples were treated with EMA prior to DNA extraction. By treating live and heat-killed Gram-positive (Streptococcus mutans) and Gram-negative (E. coli) bacteria with different concentrations of EMA, we found that EMA, at a final concentration of $100 \mu \mathrm{g} / \mathrm{ml}$, was able to effectively bind DNA from $10^{8} \mathrm{CFU} / \mathrm{ml}$ dead cells and remove the PCR signal from DNA of dead cells, without significantly affecting PCR signal from live cells. EMA cross-linking was performed as described previously [27]. Briefly, EMA (Biotium, Hayward, CA, USA) was dissolved in water to a stock concentration of $5 \mathrm{mg} / \mathrm{ml}$ and stored at $-20^{\circ} \mathrm{C}$ in the dark. EMA was added to the culture samples to a final concentration of $100 \mu \mathrm{g} / \mathrm{ml}$, and samples were incubated in the dark for $5 \mathrm{~min}$ with occasional mixing before samples were incubated on ice and light-exposed for $1 \mathrm{~min}$ using a $650-\mathrm{W}$ halogen light source placed about $20 \mathrm{~cm}$ from the samples. After photoinduced cross-linking, bacterial cells were collected by centrifugation at $5,000 \times g$ for 5 min, followed by total genomic DNA isolation.

\section{Genomic DNA Isolation}

Total genomic DNA of BHI-cultivable isolates and microbial mixture was isolated using the MasterPure ${ }^{\mathrm{TM}}$ DNA purification kit (EPICENTRE, Madison, WI, USA). Samples harvested from inter-flora competition and community integration assays were treated with EMA before total genomic DNA was isolated. DNA quality and quantity were measured by a UV spectrophotometer at 260 and $280 \mathrm{~nm}$ (Spectronic Genesys ${ }^{\mathrm{TM}}$, Spectronic Instrument, Inc. Rochester, New York, USA). 


\section{PCR-DGGE}

Amplification of bacterial 16S rRNA genes by PCR was carried out as described previously by Li et al. [21]. Briefly, the universal primer set, Bac1 and Bac2, was used to amply an approximately 300-bp internal fragment of the $16 \mathrm{~S}$ rRNA gene. A 40-nucleotide GC-clamp was added to the 5' end of the Bac1 primer. Each $50 \mu \mathrm{l}$ PCR reaction contains $100 \mathrm{ng}$ of purified genomic DNA, 40 pmol of each primer, $200 \mu \mathrm{M}$ of each dNTP, $4.0 \mathrm{mM} \mathrm{MgCl} 2,5 \mu \mathrm{l}$ of $10 \times$ PCR buffer, and $2.5 \mathrm{U}$ of Taq DNA polymerase (Invitrogen). Cycling conditions were $94^{\circ} \mathrm{C}$ for $3 \mathrm{~min}$, followed by 30 cycles of $94^{\circ} \mathrm{C}$ for $1 \mathrm{~min}, 56^{\circ} \mathrm{C}$ for $1 \mathrm{~min}$, and $72^{\circ} \mathrm{C}$ for $2 \mathrm{~min}$, with a final extension period of $5 \mathrm{~min}$ at $72^{\circ} \mathrm{C}$. The resulting PCR products were evaluated by electrophoresis in $1.0 \%$ agarose gels.

Polyacrylamide gels at an $8 \%$ concentration were prepared with a denaturing urea/formamide gradient between $40 \%$ (containing $2.8 \mathrm{~mol} / \mathrm{L}$ urea and $16 \%(\mathrm{v} / \mathrm{v})$ formamide) and $70 \%$ (containing $4.9 \mathrm{~mol} / \mathrm{L}$ urea and $28 \%$ $(v / v)$ formamide). Approximately, $300 \mathrm{ng}$ of the PCR product were applied per well. The gels were submerged in $1 \times$ TAE (Tris-Acetate-EDTA) buffer $(40 \mathrm{mmol} / \mathrm{L}$ Tris base, $40 \mathrm{mmol} / \mathrm{L}$ glacial acid acetic, $1 \mathrm{mmol} / \mathrm{L}$ EDTA), and the PCR products were separated by electrophoresis for $17 \mathrm{~h}$ at $58^{\circ} \mathrm{C}$ using a fixed voltage of $60 \mathrm{~V}$ in the Bio-Rad DCode System (Bio-Rad laboratories, Inc., Hercules, CA, USA). After electrophoresis, the gels were rinsed and stained for $15 \mathrm{~min}$ in $1 \times$ TAE buffer containing $0.5 \mu \mathrm{g} / \mathrm{ml}$ ethidium bromide, followed by $10 \mathrm{~min}$ of de-staining in $1 \times$ TAE buffer. DGGE profile images were digitally recorded using the Molecular Imager Gel Documentation system (Bio-Rad Laboratories, Hercules, CA, USA).

\section{Analysis of DGGE Profiles}

The same set of universal primers and PCR conditions were applied to all genomic DNA samples purified from cultivated microbial mixtures. The characterization of microbial flora was performed based on PCR-generated profiles of the 16S rDNA fragments on DGGE gels. Diversity Fingerprint and Diversity Database Software (BioRad), which have been widely applied in combination with the BioRad Molecular Imager Gel Documentation system to perform quantitative analysis of DGGE fingerprints, were used to assess the diversity by comparing the number of DGGE bands detected per lane. For the interflora competition and community integration assays, bands corresponding to specific bacterial species were monitored by measuring the degree of intensity at each time point and comparing with the band intensity at time zero. These analyses were only applied to evaluate relative abundance of the same species over time. Quantitative comparisons across the different species with the samples were not performed due to concerns regarding PCR amplification bias.

Identification of Most Abundant Species in the Cultivated Oral and Intestinal Microbial Flora

The most intense DNA bands were excised from the DGGE gels and transferred to a $1.5 \mathrm{ml}$ microfuge tube containing $10 \mu \mathrm{l}$ of sterile $\mathrm{ddH}_{2} \mathrm{O}$. Tubes were incubated at $4^{\circ} \mathrm{C}$ overnight before the recovered DNA samples were reamplified with the universal primer set (Bac1 and Bac2). The PCR products were then purified, sequenced, and identified as described above.

\section{Statistical Analysis}

Significance of differences between average values was analyzed by $t$ tests using MS Excel.

\section{Results}

PCR-DGGE and Sequencing Analysis Revealed Distinct Bacterial Profiles Between Cultivatable Oral and Intestinal Microbial Flora from Mice

This study aims to test the hypothesis that an established microbial community could impose selective pressure on incoming bacterial species as a way to shape and maintain the existing microbial community independent of host selection. We established an in vitro experimental system with microbial communities derived from the oral cavity and intestines of mice under the same nutritional conditions to minimize the legacy and host habitat effects. These model communities were employed to study the interactions between the oral and gut microbial floras.

The biodiversity of the BHI-cultivable microbial flora from the mice oral and intestinal samples was first assessed by PCR-DGGE analysis (Fig. 1). The banding pattern of the cultivable microbial flora from the mice oral samples was very distinct from the one displayed by the microbial community cultivated from intestinal samples (Fig. 1a, first two columns), indicating a clear difference in the composition of cultivable microbial flora between the mice oral cavity and intestinal tract. A minimum of 20 distinct bands from the oral sample and 18 bands from intestinal sample were detected.

All 300-bp 16S-rDNA amplicons resulting in predominant bands during DGGE analysis were subjected to sequence analysis and allowed identification of the corresponding bacterial species either to the genus or species level. The result, combined with full-length $16 \mathrm{~S}$ 

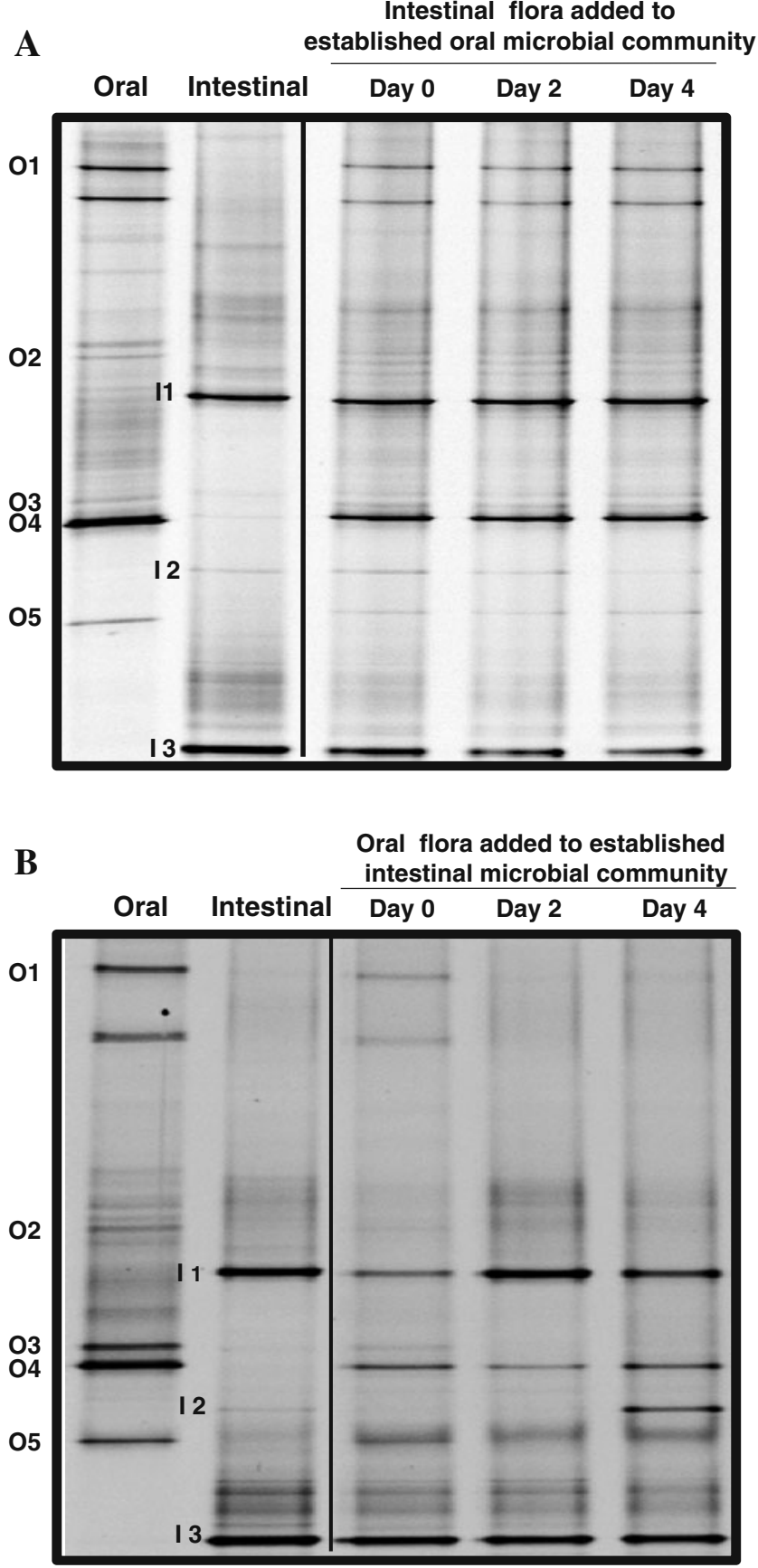

Figure 1 Community level competition between cultivable oral and intestinal microbial communities. a The oral microbial community was allowed to establish in biofilm form before intestinal bacterial mixture was added. b The intestinal microbial community was allowed to establish in pellet form for $24 \mathrm{~h}$ prior to addition of the oral bacterial mixture. Microbial profiles and changes in the banding pattern of the mixed communities were monitored by PCR-DGGE. For both setups, samples were taken every 2 days, EMA-treated, and subjected to PCR-DGGE analysis. O1-O5 represent different oral species; II-I3 represent different intestinal microbes. Two biological replicates were performed for each assay, and a representative gel image is shown
rDNA sequence data obtained from those original isolates, revealed that Streptococcus spp. (such as S. salivarius), Staphylococcus spp. (such as S. epidermidis), and Lactobacillus spp. (such as L. murinus) are among the predominant bacterial species that can be cultivated from the oral cavity of mice, while E. coli, E. faecalis, and Lactobacillus spp. (such as L. animalis) are among the abundant species that can be detected in the cultivable intestinal microflora under the experimental condition used.

The Existing Established Bacterial Communities Showed Antagonistic Effects Against Incoming Foreign Bacterial Flora

When the oral microbial mixture was allowed to establish its community (O-mix) in biofilm form prior to addition of the intestinal microbial flora, most of the major bands corresponding to oral microbial community members remained consistent in density (Fig. 1a), while the majority of the bands representing intestinal microbes, such as I2 (E. faecalis) and I3 (E. coli), displayed an obvious reduction in their density to various degrees (Fig. 1a and Supplementary Table S1). Similar results were obtained when the oral microbial community was allowed to establish itself in a cell pellet form instead of biofilm form, before intestinal microbial flora was added (data not shown).

The in vitro competition assay also revealed that the preestablished intestinal microbial community had a similar antagonistic effect toward added oral microbial flora: Most of the bands corresponding to the intestinal microbial species, such as I1 (Lactobacillus spp.), I2 (E. faecalis), and $\mathrm{I} 3$ (E. coli), did not change significantly during the experimental period up to 4 days, while most of the bands corresponding to oral bacteria, such as O1 (Staphylococcus spp.), O3 (Lactobacillus spp.), and O4 (Streptococcus spp.), were significantly reduced in their density (Fig. 1b and Supplementary Table S1).

\section{Oral and Intestinal Bacterial Isolates Are Excluded} from Communities of Foreign Origin

The results presented in Fig. 1 suggested that the incoming BHI-cultivable mice oral and intestinal microbial flora experienced an antagonistic effect by the respective preexisting foreign community. For a more detailed analysis, we performed a community integration assay tracking the survival of individual species in their original and a foreign community. DGGE analysis of the oral and intestinal communities as well as colony isolation revealed that in this study, S. salivarius and S. epidermidis are among the most frequently isolated species from the oral cavity, while E. faecalis and L. animalis are species often cultivated from the intestinal samples. These four isolates were chosen for 
testing their ability to integrate into oral and intestinal communities, and PCR-DGGE analysis was performed to monitor the status of these isolates in different communities. PCR-DGGE analysis showed that the bands corresponding to the two oral isolates remained constant in intensity throughout the experimental time period when co-cultivated with oral microbial flora, while co-cultivation with the intestinal microbial community resulted in a drastic reduction in amplicon intensity for both strains (Fig. 2a, b). A similar pattern was observed for the two intestinal isolates, which persisted quite well in the intestinal community, but experienced a significant decline when grown in the presence of oral flora (Fig. 2c, d).

In order to perform a more quantitative analysis, a colony counting approach was used for confirming bacterial community preferences. The strains $S$. salivarius 57.I and E. faecalis OG1SSp carrying kanamycin and spectinomycin antibiotic marker, respectively, were chosen as representatives of oral and intestinal bacterial species for the community integration assay. Colony counting data revealed that upon addition to the pre-existing intestinal community, S. salivarius 57.I suffered a drastic reduction in
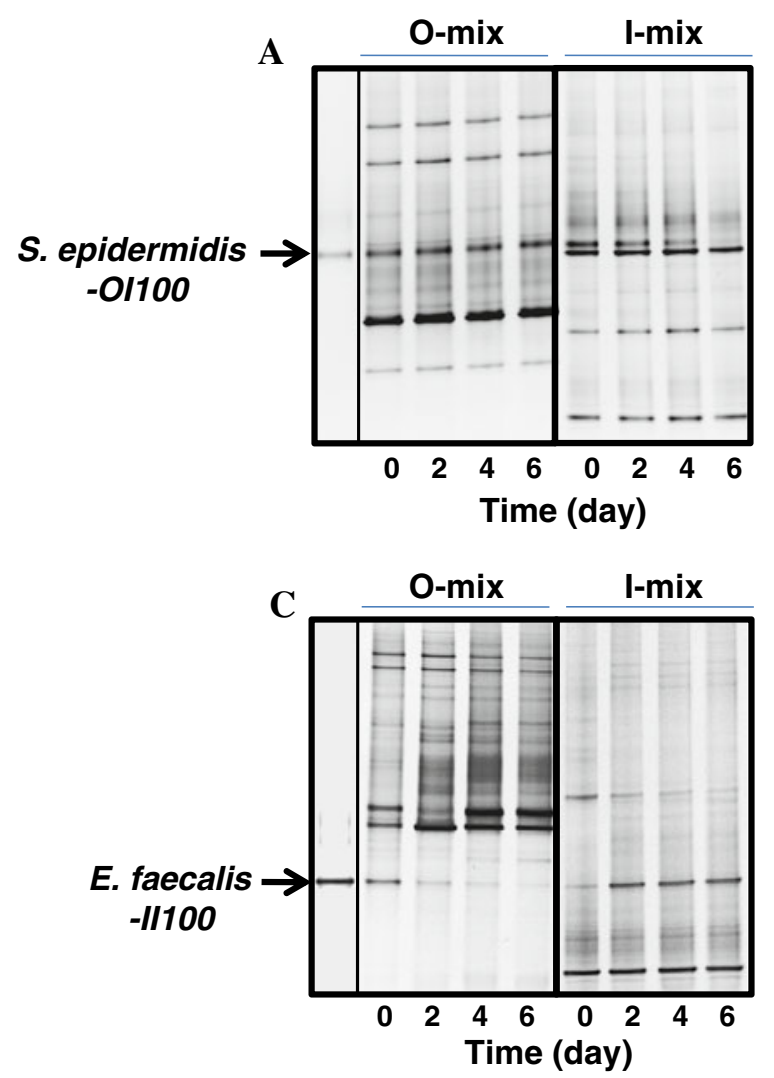

Figure 2 PCR-DGGE analysis of community integration assay. Two original oral isolates, S. epidermidis-OI100 (a) and S. salivariusOI101 (b), and two intestinal isolates, E. faecalis-II100 (c) and $L$. animalis-IIIO1 (d), were co-cultivated with either a pre-established oral or intestinal community in a 1:10 isolate-to-mixture ratio in cell its viable count (Fig. 3). Its proportion relative to the total intestinal bacteria count dropped from around 10 to less than $0.03 \%$ after 4 days of co-cultivation, while no significant change in the total bacterial count was apparent. Furthermore, no decline was evident when S. salivarius was added to the pre-existing oral biofilm community. Consistent with these results, the intestinal representative strain, E. faecalis OG1SSp, experienced a similar significant reduction in viable count in the presence of an oral community, while its population remained constant upon incubation with the intestinal community (Fig. 3).

Maximum Inhibitory Effect Toward Foreign Bacteria Required the Whole Community

In this study, we explore whether this community-based antagonistic action toward foreign bacteria requires the presence or the participation of the most of the community members or if it could be exerted by individual species within the original flora. To distinguish these possibilities, an E. faecalis strain (OG1SSp) was chosen as the representative of the intestinal community and co-
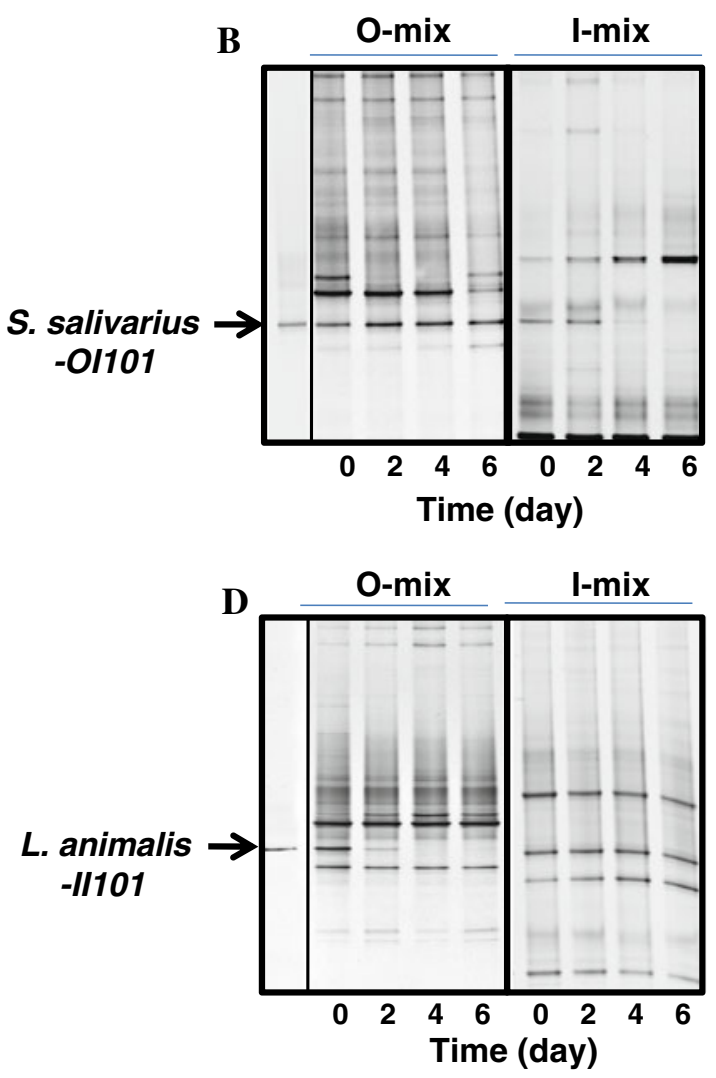

numbers. Samples were taken every 2 days, EMA-treated, and subjected to PCR-DGGE analysis to monitor the profiles of the tested strains and whole communities. Two biological replicates were performed for each assay, and a representative gel image is shown 


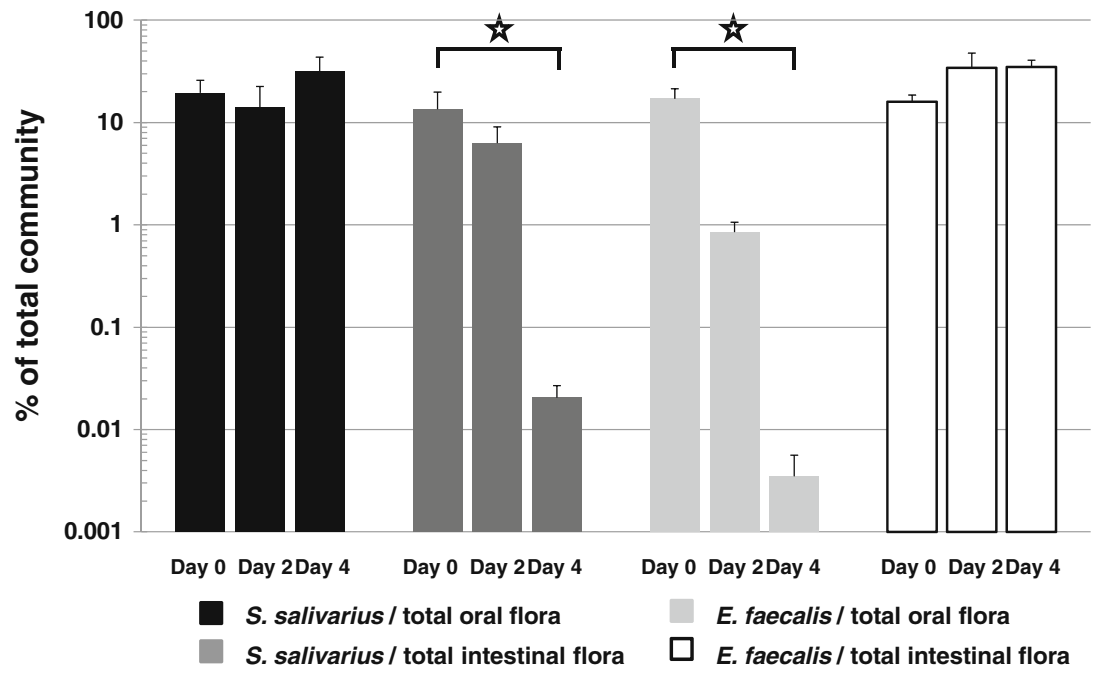

Figure 3 Viable count analysis of community integration assay. Oral and intestinal representatives, S. salivarius-57.I and E. faecalisOG1SSp, were co-cultivated with the oral (O-mix) and intestinal (I-mix) communities in a 1:10 isolate-to-community ratio in cell numbers. The viability of the tested strains and the whole oral and intestinal community was monitored every $48 \mathrm{~h}$ up to 4 days. The data were expressed as percentage of viable count of tested strains to the total viable count of the pre-established communities. Black bar

cultivated in a 1:10 ratio with oral biofilms derived from either single oral isolates, a subset (mixture of four oral isolates), or the whole cultivable oral flora (Fig. 4). The viability of $E$. faecalis and the respective oral community members of the represents the percentage of $S$. salivarius/total oral community counts; dark gray bar represents the percentage of $S$. salivarius/total intestinal community count; light gray bar represents the percentage of $E$. faecalis/total oral community count; and white bar represents the percentage of $E$. faecalis/total intestinal community count. Three replicates were performed for each viability count assay. Average values \pm SD are shown. Star indicates that the value obtained for this time point is significantly lower than at day $0(P<0.05)$

different test conditions were monitored via CFU determination. The subset of oral microbial flora exerted much stronger inhibition on E. faecalis than individual oral isolates, and the maximum antagonistic effect was observed

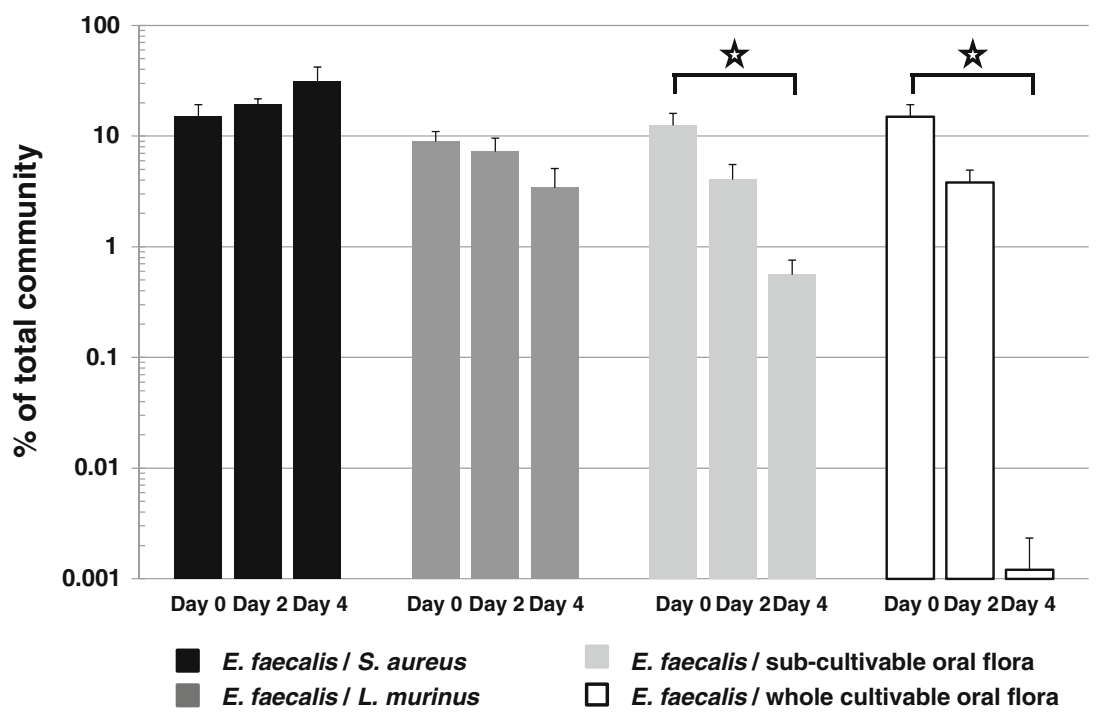

Figure 4 Inhibitory effect of oral communities with different biodiversity on the intestinal representative strain, E. faecalisOG1SSp. E. faecalis-OG1SSp was co-cultivated with biofilms of single oral isolates ( $S$. aureus or L. murinus), a subset of cultivable oral flora including $S$. aureus, L. murinus, S. salivarius, and $S$. epidermidis, or the whole cultivable oral microbial flora at a 1:10 ratio in cell number. The viability count of the $E$. faecalis strain and total oral bacterial cells was monitored every $48 \mathrm{~h}$. The data were expressed as percentage of viable count of E. faecalis in respect to the total viable count of oral bacteria. Black bar represents the percentage of $E$. faecalis/S. aureus counts; dark gray bar represents the percentage of E. faecalis/L. murinus count; light gray bar represents the percentage of E. faecalis/sub-oral flora count; and white bar represents the percentage of E. faecalis/whole oral community count. Three replicates were performed for each viability count assay. Average values \pm SD are shown. Star indicates that the value obtained for this time point is significantly lower than at day $0(P<0.05)$ 
when $E$. faecalis was co-cultivated with the whole cultivable oral flora (Fig. 4). Similar results were obtained for $S$. salivarius 57.I, an oral representative strain carrying a kanamycin resistance marker, which suffered the most drastic reduction in its viable count when co-cultivated with the whole cultivable intestinal community (Fig. 5).

Spent Medium of Oral and Intestinal Microbial Communities Did Not Have a Killing Effect on Bacterial Isolates of Foreign Origin

The bacterial community integration assay showed that all the tested bacterial isolates were able to integrate into their own original community, while suffering inhibitory effects when grown in the presence of foreign microbial flora. One probable explanation for the observed inhibitory effect could be simply due to the accumulation of certain toxic metabolites released by the community during its growth. To test this possibility, the original oral isolate $S$. salivariusOI101 and the intestinal isolate E. faecalis-II100 were inoculated into the spent media of oral and intestinal communities, and their viability was monitored every $48 \mathrm{~h}$ over a 4-day time period. Both strains persisted well in the spent media of both oral and intestinal flora throughout the experimental period (Supplemental Fig. 1) indicating that the individual microflora did not accumulate toxic metabolites under the test condition.

\section{Discussion}

Extensive studies have been performed to reveal the microbial diversity in human oral cavity and intestinal tract, two important microbial habitats along the human GI tract $[1,12,30,42]$. So far, more than a thousand distinct species or phylotypes have been recovered from the human intestinal tract [31, 41, 42], while over 700 species have been identified from human oral cavity based on traditional cultural studies and culture-independent molecular studies $[1,29,30]$. Even though both the oral cavity and the intestinal tract are part of the human GI tract and most intestinal microbes may initially enter through the oral cavity, recent microbial community analysis showed that the two habitats share surprisingly few bacterial species $[25,29,31]$. This raised an interesting and fundamental question: How are these peculiar microbial communities established and what are the underlying mechanisms in maintaining the existing microflora?

A critical role for host factors in shaping the GI tractassociated endogenous microbial communities has previously been implicated [19]. Reciprocal gut microflora transplants from Zebrafish and mice to germ-free recipients by Rawls et al. established that the transplanted microfloras are similar to the community of origin [33]. However, the relative richness of the lineages shifted toward the composition of the normal intestinal microflora in the

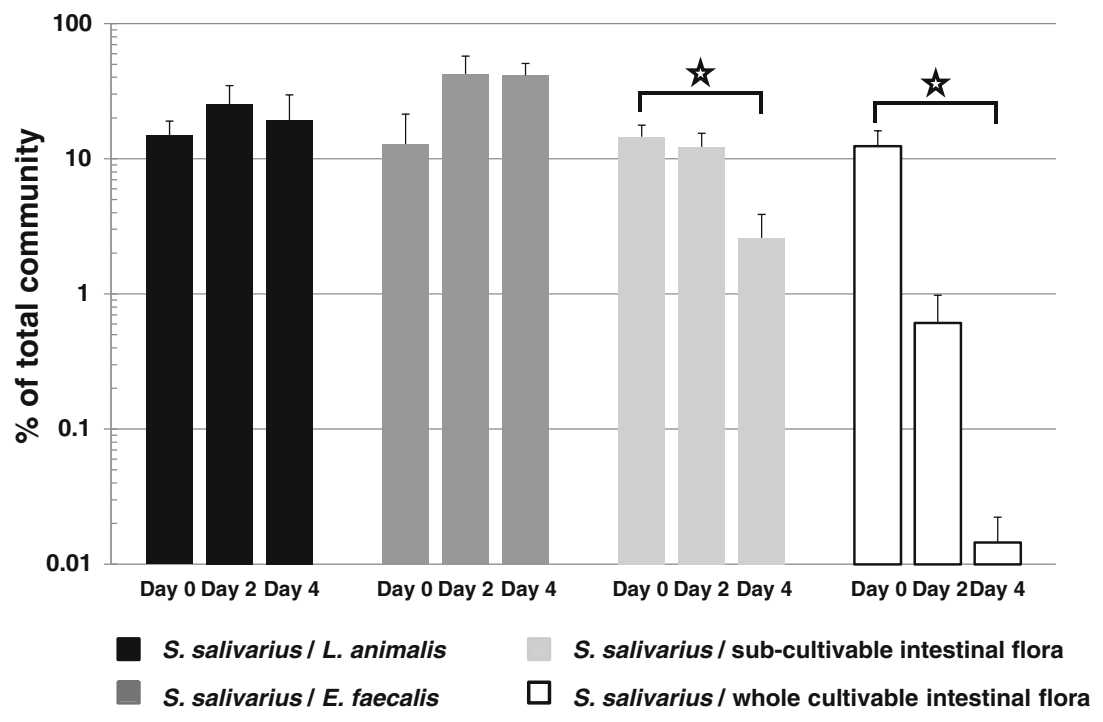

Figure 5 Inhibitory effect of intestinal communities with different biodiversity on the oral representative strain, S. salivarius-57.I. S. salivarius strain was co-cultivated with a pelleted community including either single intestinal isolates ( $L$. animalis and $E$. faecalis), a subset of cultivable intestinal flora including $L$. animalis, E. faecalis, $E$. coli, and B. caccae, or the whole cultivable intestinal microbial flora at a 1:10 ratio in cell numbers. The viability count of $S$. salivarius and total intestinal bacteria community was monitored every $48 \mathrm{~h}$. The data were expressed as percentage of viable count of
S. salivarius in respect to the total viable count of intestinal bacteria. Black bar represents the percentage of $S$. salivarius/L. animalis counts; dark gray bar represents the percentage of $S$. salivarius/E. faecalis count; light gray bar represents the percentage of $S$. salivarius/sub-intestinal flora count; and white bar represents the percentage of $S$. salivarius/whole intestinal community count. Three replicates were performed for each viability count assay. Average values \pm SD are shown. Star indicates that the value obtained for this time point is significantly lower than at day $0(P<0.05)$ 
recipient, thus convincingly demonstrating the host habitat selection on the intestinal tract-associated microbial community [33]. The same principle could apply to the microbiota associated with different parts of the human GI tract. Different micro-environments along the GI tract (local anatomical structures, nutrient availability, and host immune reaction) could exert selective pressure on the microbes which result in the distinct microbial communities.

While the local host and nutritional factors could contribute significantly to the microbial compositions in different parts of the GI tract, we hypothesized that the existing microbial flora plays a role in selecting the bacterial species that can be integrated into the community independent of host factors. To test this hypothesis, we established an in vitro system comprised of cultivable microbial flora isolated from two distinct niches along the GI tract of mice, the oral cavity and intestinal tract. One of the limitations of studying microbial community using culture-based methods is the often underrepresented microbial composition in cultivable microbes compared to the original samples [38]. Due to this limitation, the in vitro system we established in this study using BHIcultivable microbial species can only represent small subsets of the two distinct microbiotas along the mice GI tract. Nevertheless, culture-dependent methods are indispensable in providing valuable information, including both phenotypic and genetic characterization of individual bacteria, as well as new physiological functions prompted by the interactions among different microbial community inhabitants. Our in vitro study using two cultivable microbial sub-communities allowed a glimpse into the intriguing dynamic interactions between the microbial floras associated with oral cavity and intestinal tract of mice.

16S rDNA-based PCR-DGGE analysis revealed distinct microbial patterns within the two cultivable communities (Fig. 1). Staphylococcus spp., Streptococcus spp., and Lactobacillus spp. are among the most dominant genera recovered from the oral samples of mice, while Enterococcus spp., E. coli, and Lactobacillus spp. were most frequently cultivated from the corresponding intestinal tracts. These results correlated well with previous cultivation-based microbial community analyses of the GI tract-associated microbiota of mice [4, 34].

The in vitro community competition assay revealed an intriguing antagonistic action between cultivable oral and intestinal sub-floras. Depending on the sequence of inoculation, the established pre-existing microbial community always exhibited a survival advantage and exerted inhibitory effects on the members of the incoming flora of different origin (Fig. 1). In environmental ecosystems, community level competition is a relatively rare event and only occurs when natural barriers break down [40]. However, when this happens, one community may overtake a second community, causing all or most of its members to go extinct [40]. Furthermore, pre-existing communities develop invasion resistance over time, which enhances their ability to defend their habitats $[6,35]$. The behavior of the two distinct communities tested in this study is consistent with these observations. The original isolates and their closely related strains tested persisted well in their original community and were inhibited when grown together with the microbial community of different origin (Figs. 2 and 3). In environmental ecology, it has been shown that isolated communities often interact by sending "invasion propagules" between one another. Such smallscale probes, however, often had no permanent effect on the communities due to the invasion resistance developed in the communities and were regarded as one of the mechanisms in maintaining the stability of the community $[6,35]$. Although most of the current invasion resistance concepts are derived from non-microbial ecosystem, the same principle could apply to the microbial world. Our in vitro data are in agreement with above ecological phenomenon, suggesting that the dynamics among members from the same community could contribute to the invasion resistance, thus play an important role in maintaining the structure and stability of the community.

The maximum inhibitory effect toward foreign bacteria was achieved when the whole cultivable community was used, suggesting a community-based antagonistic action (Figs. 4 and 5). This is in agreement with observation in other ecosystems where species-rich communities are more resistant to invasion by exotics than species-poor communities [13]. Our in vitro oral and intestinal community comprised of at least 20 and 18 different bacterial species, respectively. The members derived from the same microflora are likely to have co-evolved and therefore have experienced extensive interactions with each other and built certain coordinated relationships among themselves [17]. This might contribute to the community invasion resistance by initiating a series of defense responses through synergistic interactions among the endogenous residents when foreign bacterial invaders are encountered. Our observation is corroborated by similar phenomena that have been reported for other microbial communities. The microbial community isolated from the surface of the marine alga Ulva australis was demonstrated to interact synergistically in biofilms and resist foreign bacterial invasion to a greater extent than single-species biofilms [5]. Furthermore, the result of the spent medium assay indicates that the antagonistic effects toward foreign bacteria are unlikely due to the accumulation of toxic metabolites from the respective communities (Supplementary Fig. S1) but rather the result of more active processes involving interactions between foreign bacteria and community members.

Clearly, this newly established inter-flora interaction system is just a starting point and far from being a perfect 
system. Since only a small sub-population of oral and intestinal communities can be recovered by cultivation, the data obtained in this study cannot entirely represent the real situation in the host. Nevertheless, we were able to consistently observe community-based antagonistic actions toward foreign bacteria even under the same nutritional conditions independent of host environment. Similar results were obtained for another major intestinal representative species $E$. coli (see the accompanying manuscript $\mathrm{He}$ et al. 2010). Taking advantage of the well-understood genetic background and pre-established mutant collections in $E$. coli, we were able to reveal the molecular mechanism underlying the observed phenomenon.

From an evolutionary and ecological point of view, the GI tract represents a complex ecosystem with several distinct microbial habitats, such as the oral cavity and intestinal tract. The microbial diversity within each habitat is the result of co-evolution between microbial communities and their specific host factors, and the actual events of community establishment and maintenance often involve a series of microbial intra-, interspecies, and microbe-host interactions. While host factors could be the key determinants for establishing the GI tract-associated microbial community structure, our in vitro analyses of cultivable mice oral and intestinal microbial communities suggested an important role of the dynamics within a given microbial flora in shaping and maintaining the existing communities. The observed inhibitory effect toward foreign bacteria could contribute to the protective/probiotic effect of established microbial communities within the GI tract and play a significant role in fending off potential pathogens which would be recognized as foreign.

Acknowledgments We thank Z. Yang for their help in the initial characterization of mice GI tract isolates, L. Li, W. Hu, and J. Zhou for discussions, and R. Burne and G. Dunny for providing bacterial strains. The study was supported in part by NIH GM54666 to WS.

Open Access This article is distributed under the terms of the Creative Commons Attribution Noncommercial License which permits any noncommercial use, distribution, and reproduction in any medium, provided the original author(s) and source are credited.

\section{References}

1. Aas JA, Paster BJ, Stokes LN, Olsen I, Dewhirst FE (2005) Defining the normal bacterial flora of the oral cavity. J Clin Microbiol 43:5721-5732

2. Beck J, Garcia R, Heiss G, Vokonas PS, Offenbacher S (1996) Periodontal disease and cardiovascular disease. J Periodontol 67:1123-1137

3. Berbari E, Cockerill F III, Steckelberg J (1997) Infective endocarditis due to unusual or fastidious microorganisms. Mayo Clin Proc 72:532-542
4. Blais J, Lavoie M (1990) Effect of dietary components on the indigenous oral bacterial flora of BALB/c mice. J Dent Res 69:868873

5. Burmolle M, Webb JS, Rao D, Hansen LH, Sorensen SJ, Kjelleberg S (2006) Enhanced biofilm formation and increased resistance to antimicrobial agents and bacterial invasion are caused by synergistic interactions in multispecies biofilms. Appl Environ Microbiol 72:3916-3923

6. Case T (1990) Invasion resistance arises in strongly interacting species-rich model competition communities. Proc Natl Acad Sci USA 87:9610-9614

7. Cebra JJ (1999) Influences of microbiota on intestinal immune system development. Am J Clin Nutr 69:1046S-1051

8. Chen YM, Feng CW, Chiu CF, Burne RA (2008) cadDX operon of Streptococcus salivarius 57.I. Appl Environ Microbiol $74: 1642-1645$

9. Chuang ON, Schlievert PM, Wells CL, Manias DA, Tripp TJ, Dunny GM (2009) Multiple functional domains of Enterococcus faecalis aggregation substance Asc10 contribute to endocarditis virulence. Infect Immun 77:539-548

10. Dahlen G (1993) Role of suspected periodontopathogens in microbiological monitoring of periodontitis. Adv Dent Res $7: 163-174$

11. Dodman T, Robson J, Pincus D (2000) Kingella kingae infections in children. J Paediatr Child Health 36:87-90

12. Eckburg PB, Bik EM, Bernstein CN, Purdom E, Dethlefsen L, Sargent M, Gill SR, Nelson KE, Relman DA (2005) Diversity of the human intestinal microbial flora. Science 308:1635-1638

13. Elton CS (1958) The ecology of invasions by plants and animals. Chapman and Hall, London

14. Guarner F, Malagelada J (2003) Gut flora in health and disease. Lancet 361:512-519

15. Hugot J (2004) Inflammatory bowel disease: a complex group of genetic disorders. Best Pract Res Clin Gastroenterol 18:451-462

16. Kreth J, Justin M, Shi W, Qi F (2005) Co-ordinated bacteriocin production and competence development: a possible mechanism for taking up DNA from neighbouring species. Mol Microbiol 57:392404

17. Kuramitsu HK, He X, Lux R, Anderson MR, Shi W (2007) Interspecies interactions within oral microbial communities. Microbiol Mol Biol Rev 71:653-670

18. Lederberg J (2000) Infectious history. Science 288:287-293

19. Ley R, Peterson D, Gordon J (2006) Ecological and evolutionary forces shaping microbial diversity in the human intestine. Cell 124:837-848

20. Ley R, Backhed F, Turnbaugh P, Lozupone CA, Knight RD, Gordon JI (2005) Obesity alters gut microbial ecology. Proc Nati Acad Sci 102:11070-11075

21. Li Y, Ku CYS, Xu J, Saxena D, Caufield PW (2005) Survey of oral microbial diversity using PCR-based denaturing gradient gel electrophoresis. J Dent Res 84:559-564

22. Mangin I, Bonnet R, Seksik P, Rigottier-Gois L, Sutren M, Bouhnik Y, Neut C, Collins MD, Colombel JF, Marteau P, Dore J (2004) Molecular inventory of faecal microflora in patients with Crohn's disease. FEMS Microbiol Ecol 50:25-36

23. Marsh P (1994) Microbial ecology of dental plaque and its significance in health and disease. Adv Dent Res 8:263-271

24. Martin-Laurent F, Philippot L, Hallet S, Chaussod R, Germon JC, Soulas G, Catroux G (2001) DNA Extraction from soils: old bias for new microbial diversity analysis methods. Appl Environ Microbiol 67:2354-2359

25. Maukonen J, Matto J, Suihko ML, Saarela M (2008) Intraindividual diversity and similarity of salivary and faecal microbiota. J Med Microbiol 57:1560-1568

26. Nishihara T, Koseki T (2004) Microbial etiology of periodontitis. Periodontology 2000 36:14-26 
27. Nocker A, Camper AK (2006) Selective removal of DNA from dead cells of mixed bacterial communities by use of ethidium monoazide. Appl Environ Microbiol 72:1997-2004

28. Palmer C, Bik E, DiGiulio D, Relman D, Brown P (2007) Development of the human infant intestinal microbiota. PLoS Biol 5:1556-1573

29. Paster B, Olsen I, Aas JA, Dewhirst FE (2006) The breadth of bacterial diversity in the human periodontal pocket and other oral sites. Periodontology 2000 42:80-87

30. Paster BJ, Boches SK, Galvin JL, Ericson RE, Lau CN, Levanos VA, Sahasrabudhe A, Dewhirst FE (2001) Bacterial diversity in human subgingival plaque. J Bacteriol 183:3770-3783

31. Rajilic-Stojanovic M, Smidt H, de Vos W (2007) Diversity of the human gastrointestinal tract microbiota revisited. Environ Microbiol 9:2125-2136

32. Rakoff-Nahoum S, Paglino J, Eslami-Varzaneh F, Edberg S, Medzhitov R (2004) Recognition of commensal microflora by tolllike receptors is required for intestinal homeostasis. Cell 118:229-241

33. Rawls JF, Mahowald MA, Ley RE, Gordon JI (2006) Reciprocal gut microbiota transplants from Zebrafish and Mice to germ-free recipients reveal host habitat selection. Cell 127:423-433

34. Rodrigue L, Lavoie M (1996) Comparison of the proportions of oral bacterial species in BALB/c mice from different suppliers. Lab Anim 30:108-113
35. Roughgarden J (1987) Community coevolution: a comment. Evolution 41:1130-1134

36. Socransky S (1979) Criteria for the infectious agents in dental caries and periodontal disease. J Clin Periodontol 6:16-21

37. Sonnenburg J, $\mathrm{Xu} \mathrm{LJ}$, Leip $\mathrm{DD}$, Chen $\mathrm{CH}$, Westover BP, Weatherford J, Buhler JD, Gordon JI (2005) Glycan foraging in vivo by an intestine-adapted bacterial symbiont. Science 307:1955-1959

38. Staley JT, Konopka A (1985) Measurement of in situ activities of nonphotosynthetic microorganisms in aquatic and terrestrial habitats. Annu Rev Microbiol 39:321-346

39. Tanner ACR, Paster BJ, Lu SC, Kanasi E, Kent R Jr, Van Dyke T, Sonis ST (2006) Subgingival and tongue microbiota during early periodontitis. J Dent Res 85:318-323

40. Vermeij $G$ (1991) When biotas meet: understanding biotic interchange. Science 253:1099-1104

41. Wang M, Ahrne S, Jeppsson B, Molin G (2005) Comparison of bacterial diversity along the human intestinal tract by direct cloning and sequencing of 16S rRNA genes. FEMS Microbiol Ecol 54:219-231

42. Wang X, Heazlewood SP, Krause DO, Florin THJ (2003) Molecular characterization of the microbial species that colonize human ileal and colonic mucosa by using 16S rDNA sequence analysis. J Appl Microbiol 95:508-520 